\title{
Ultralow-threshold erbium-implanted toroidal microlaser on silicon
}

\author{
A. Polman ${ }^{a}$ \\ Department of Applied Physics, California Institute of Technology, Pasadena, California 91125 \\ and FOM-Institute AMOLF, Kruislaan 407, 1098 SJ Amsterdam, The Netherlands \\ B. Min \\ Department of Applied Physics, California Institute of Technology, Pasadena, California 91125 \\ J. Kalkman \\ FOM-Institute AMOLF, Kruislaan 407, 1098 SJ Amsterdam, The Netherlands
}

\author{
T. J. Kippenberg and K. J. Vahalab) \\ Department of Applied Physics, California Institute of Technology, Pasadena, California 91125
}

(Received 6 November 2003; accepted 15 December 2003)

\begin{abstract}
We present an erbium-doped microlaser on silicon operating at a wavelength of $1.5 \mu \mathrm{m}$ that operates at a launched pump threshold as low as $4.5 \mu \mathrm{W}$. The $40 \mu \mathrm{m}$ diameter toroidal microresonator is made using a combination of erbium ion implantation, photolithography, wet and dry etching, and laser annealing, using a thermally grown $\mathrm{SiO}_{2}$ film on a $\mathrm{Si}$ substrate as a starting material. The microlaser, doped with an average Er concentration of $2 \times 10^{19} \mathrm{~cm}^{-3}$, is pumped at $1480 \mathrm{~nm}$ using an evanescently coupled tapered optical fiber. Cavity quality factors as high as $3.9 \times 10^{7}$ are achieved, corresponding to a modal loss of $0.007 \mathrm{~dB} / \mathrm{cm}$, and single-mode lasing is observed. (c) 2004 American Institute of Physics. [DOI: 10.1063/1.1646748]
\end{abstract}

The design and fabrication of a silicon-based laser, integrated with optical and electronic components on a single chip, is one of the main challenges in silicon-based optoelectronic technology. Such a laser must have small size, low pump threshold, and must be integrated with fiber and/or planar waveguide architectures on $\mathrm{Si}$. Erbium-doped $\mathrm{SiO}_{2}$ is an ideal gain medium for such a laser, as $\mathrm{SiO}_{2}$ is a low-loss waveguide medium and $\mathrm{Er}^{3+}$ ions show optical transitions around $1.5 \mu \mathrm{m}$, the standard optical telecommunication wavelength. Recently, a new technique was developed to fabricate ultrahigh quality $(Q) \mathrm{SiO}_{2}$ microresonators on $\mathrm{Si}^{1}$ that have cavity quality factors as high as $10^{8}$. When coated with erbium using a sol-gel technique ${ }^{2}$ these resonators show optical gain at $1.5 \mu \mathrm{m}$. However, the sol-gel process is not compatible with standard $\mathrm{Si}$ fabrication processing, and has limited control over Er depth profile and concentration. Also, the coupling conditions that lead to optimized gain have not been studied. In this paper, we use erbium ion implantation, ${ }^{3}$ a standard Si processing technique, in combination with the ultrahigh $Q$ cavity fabrication technique to fabricate a $\mathrm{Si}$ based microlaser operating at $1.5 \mu \mathrm{m}$. By optimizing the coupling distance between the tapered pump fiber and the resonator we are able to observe single-mode lasing at a launched power pump threshold as low as $4.5 \mu \mathrm{W}$, much lower than any Er-doped laser. We propose several applications of the combination of ion implantation with the microresonator geometry.

Erbium-doped silica toroidal resonators on $\mathrm{Si}$ were fabricated using a combination of ion implantation, optical lithography, etching and laser annealing. First, an intrinsic $\mathrm{Si}(100)$ wafer with a $2 \mu \mathrm{m}$ thick thermally grown $\mathrm{SiO}_{2}$ film

\footnotetext{
${ }^{a)}$ Electronic mail: polman@amolf.nl

b)Electronic mail: vahala@caltech.edu
}

was implanted with $2 \mathrm{MeV}$ Er ions to a total fluence of 4.2 $\times 10^{15} \mathrm{~cm}^{-2}$. The implantation leads to a Gaussian Er depth profile peaking at a depth of $560 \mathrm{~nm}$, at a peak concentration of 0.35 at. \%. To optically activate the Er, the implanted wafer was annealed at $800^{\circ} \mathrm{C}$ for $60 \mathrm{~min}$. in vacuum. Next, circular disks with diameters in the range $40-100 \mu \mathrm{m}$ were defined in the Er-implanted oxide using standard photolithography and etching. These were then isolated from the $\mathrm{Si}$ substrate using isotropic dry etching in $\mathrm{XeF}_{2}$, leaving an erbium-doped silica disk suspended by a circular $\mathrm{Si}$ pillar. Finally, the microdisks were irradiated with a $\mathrm{CO}_{2}$ laser beam $(\lambda=10.6 \mu \mathrm{m})$. As has been demonstrated before, ${ }^{1}$ this leads to melting and subsequent collapsing of the silica disk to a toroidal shape, with limiting dimensions defined by the width of the Si support pillar. Insets in Fig. 1 show scanning electron microscopy images of the planar microdisk as well as the toroidal resonator after laser annealing.

Figure 1 shows photoluminescence spectra of the Erimplanted disk before and after laser-annealing, taken using optical excitation at $488 \mathrm{~nm}$ under normal incidence. A spectrum that is characteristic for intra- $4 f$ emission of $\mathrm{Er}^{3+}$ in $\mathrm{SiO}_{2}$ is clearly observed. Luminescence decay traces at $1.535 \mu \mathrm{m}$ are shown in the inset of Fig. 1 for both disk and toroid, and show similar lifetimes of 10-12 ms. This implies that the local environment around the implanted Er ions is not affected by the laser annealing process.

To couple light in and out of the Er-implanted toroid's whispering gallery mode, a tapered optical fiber was aligned in the toroid's equatorial plane. ${ }^{4}$ The fiber was attached to a piezoelectric stage which allows for precise positioning of the taper with respect to the toroid. The air gap between taper and toroid was varied in $20 \mathrm{~nm}$ steps over the range $0-1200$ $\mathrm{nm}$. In this geometry, the details of which have been studied recently ${ }^{5}$ the evanescent field of the taper couples to a resonator mode, the coupling efficiency being determined by the 


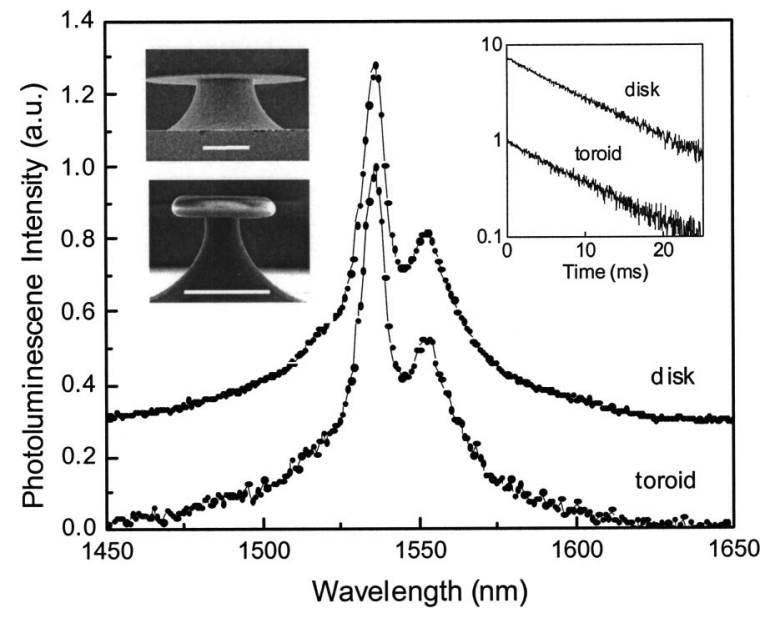

FIG. 1. Photoluminescence spectra of Er-implanted microresonators before and after laser annealing (the spectrum for the disk is shifted vertically for clarity). The right inset shows photoluminescence decay traces measured at $\lambda=1535 \mathrm{~nm}$ for both a disk and a toroid. The left insets show scanning electron microscopy images of a microresonator disk and toroid. Scale bars are both $43 \mu \mathrm{m}$.

gap width and the degree of phase matching. At the same time, the fiber taper serves to couple lasing power from the resonator. A single-frequency tunable external-cavity diode laser operating around $1480 \mathrm{~nm}$ was used to pump the ${ }^{4} I_{15 / 2} \rightarrow{ }^{4} I_{13 / 2}$ transition in $\mathrm{Er}^{3+}$ and a spectrum analyzer was used to measure any transmitted pump as well as the lasing spectrum at the fiber output.

The quality factor of the Er-implanted toroids determined from the spectral width of a transmission resonance around $\lambda=1411 \mathrm{~nm}$ (i.e., well outside the $\mathrm{Er}$ absorption band) was $Q=3.9 \times 10^{7}$ for the best resonators. This demonstrates that ion implantation is well compatible with the planar micro-resonator fabrication process. Indeed, the value for $Q$ found here translates to a modal propagation loss of the excited toroidal mode as low as $0.007 \mathrm{~dB} / \mathrm{cm}$.

The inset in Fig. 2 shows a lasing spectrum of an Erimplanted toroid pumped at $1465 \mathrm{~nm}$. Single-mode lasing is observed at $1557.03 \mathrm{~nm}$. At a given pump power, the lasing wavelength depends on the coupling conditions. If the optical fiber is weakly coupled to the resonant mode, gain tran-

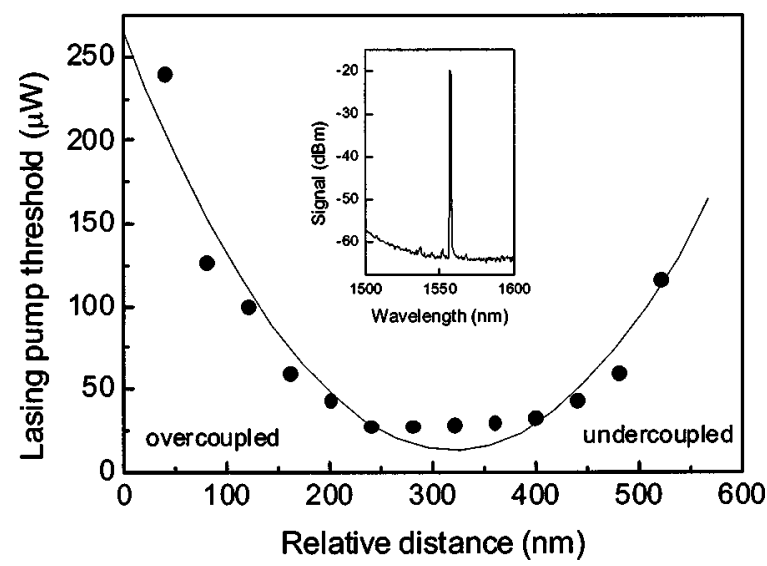

FIG. 2. Launched pump power threshold for lasing as a function of relative coupling gap distance between fiber taper and Er-implanted microtoroid (diameter $45 \mu \mathrm{m}$ ). $\lambda_{\text {pump }}=1480 \mathrm{~nm}, \lambda_{\text {lasing }}=1578 \mathrm{~nm}$. The drawn line is a guide for the eye. The inset shows a single-mode lasing spectrum measured

resonator we find that the optimum Er concentration for
under $1465 \mathrm{~nm}$ pumping.

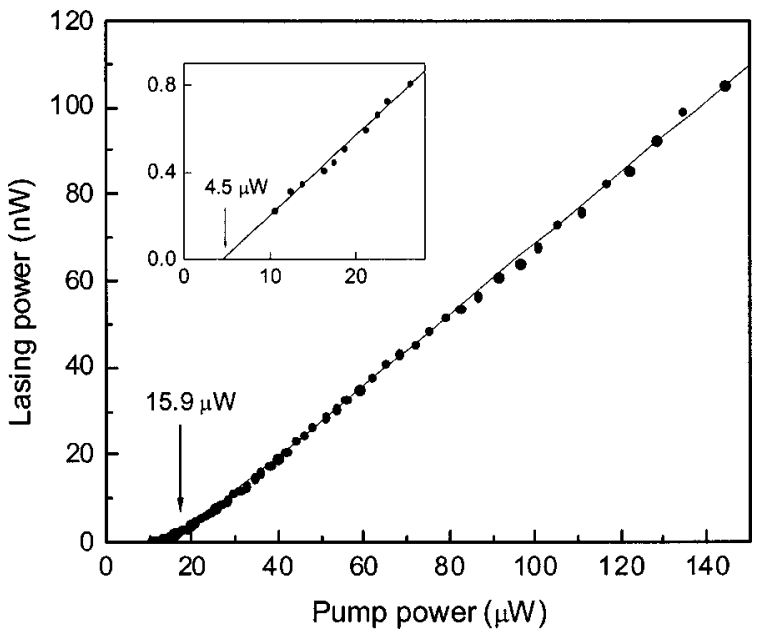

FIG. 3. Unidirectional lasing power as a function of launched power for a $32 \mu \mathrm{m}$ diameter Er-implanted microtoroid. The lasing threshold is $15.9 \mu \mathrm{W}$. the best resonator, showing a pump threshold of $4.5 \mu \mathrm{W}$.

sitions are favored between the highest Stark levels of the ground manifold and the lowest levels in the first excited state manifold, leading to gain at the long wavelength side of the spectrum. At smaller coupling gaps and thus stronger waveguide-resonator coupling the main lasing transition shifts to progressively shorter-wavelength modes. Detailed measurements of this effect and modeling that accurately reproduces the found trends will be published elsewhere. ${ }^{6}$

To further show the relation between coupling conditions and optical gain characteristics, Fig. 2 shows the lasing threshold as a function of gap width between the fiber taper and the toroid. The distance was varied over a range of 500 $\mathrm{nm}$ around a central gap distance of $\sim 800 \mathrm{~nm}$. In the undercoupled regime, with the taper far away from the resonator, a lasing threshold of $\sim 100 \mu \mathrm{W}$ is observed. Reducing the gap width, power is more efficiently coupled in the resonator and the threshold reduces to a value of $\sim 25 \mu \mathrm{W}$ for this particular resonator. Then, as the gap distance is further reduced, the strong over-coupling reduces the cavity's $Q$ and thus the threshold increases. Note that both in the under-coupled and over-coupled cases the actual power coupled into the resonator is much lower than the launched power.

Figure 3 shows a measurement of lasing power as a function of launched input power, measured at an optimized coupling distance. The lasing threshold is $15.9 \mu \mathrm{W}$ and the differential quantum efficiency above threshold is $0.16 \%$ (taking into account bidirectional lasing). The power absorbed in the resonator at minimum threshold (measured by monitoring the transmitted pump power) is $11.7 \mu \mathrm{W}$. The inset shows a measurement taken on a different resonator, taken over a less extended power range than in the main figure. It shows a lasing threshold as low at $4.5 \mu \mathrm{W}$ (absorbed power 2 to $3 \mu \mathrm{W}$ ).

To obtain further insight in the threshold characteristics of these Er-implanted toroidal resonators, a model was developed that solves the rate equations governing inversion and amplification in the pumped resonator. Assuming an intrinsic $Q=2 \times 10^{7}$, a toroid diameter of $50 \mu \mathrm{m}$, an Er emission lifetime of $10 \mathrm{~ms}$, and optimal coupling of the taper and resonator we find that the optimum Er concentration for $\lambda_{\text {pump }}=1465 \mathrm{~nm}, \lambda_{\text {lasing }}=1558 \mathrm{~nm}$. The inset shows a measurement made on 
achieving the lowest threshold is $2 \times 10^{19} \mathrm{~cm}^{-3}$, close to the value chosen in this work, assuming all $\mathrm{Er}$ has homogeneously spread through the resonator. For this concentration the calculated minimum lasing threshold under optimized (slightly under-coupled) conditions is $2.0 \mu \mathrm{W}$. This value is close to the lowest minimum threshold found here experimentally. Given the high quality factor of the resonators, this lowest achievable threshold is fully determined by the power required to invert the $\mathrm{Er}^{3+}$ ions, and not the cavity loss. This implies that a further reduction in threshold may be achieved by reducing the toroidal mode volume: For toroid diameters below $35 \mu \mathrm{m}$ a sub- $\mu \mathrm{W}$ threshold is predicted. The linear increase of lasing power with pump power above threshold observed in Fig. 3 is also in agreement with theory. The differential quantum efficiency is a very sensitive function of the coupling distance. ${ }^{7}$ Indeed, by changing the coupling conditions, a bi-directional output power as high as $39 \mu \mathrm{W}$ was achieved, corresponding to a slope efficiency of $10 \%$. Modeling results indicate that quantum efficiencies well above $15 \%$ can be achieved by using higher Er concentrations.

The observed ultra-low threshold for these Er-implanted microtoroid resonators is an improvement by more than an order of magnitude over the thresholds found in earlier work on bulk Er-doped microspheres ${ }^{8,9}$ or sol-gel coated microtoroids. ${ }^{2}$ This is the result of optimization of the coupling conditions, and the use of an optimized Er doping profile. It demonstrates that the use of ion implantation is not only an advantage due to it being a core silicon integrated circuit processing technology, but it also leads to the required erbium doping concentrations, depth distribution and extremely low cavity loss.

Various novel opto-electronic integration concepts involving these ultra-low threshold microlasers on silicon come to mind. One issue involves the fiber coupling, which is a true advantage as it naturally integrates an on-chip device with an external fiber communication network. Alternatively, the coupling fiber may be replaced by an on-chip waveguide that integrates the laser with an array of optical components on the chip. By co-doping the Er-implanted toroid with Si nanocrystals that act as sensitizers for $\mathrm{Er}^{10}{ }^{10}$ the pump laser could be replaced altogether, substituting it by a broad-band pump source that can be integrated on the same chip.

In conclusion, we have fabricated an Er-doped toroidal microlaser with a lasing threshold of $4.5 \mu \mathrm{W}$. The fabrication process is fully compatible with $\mathrm{Si}$ integrated circuit processing technology, and results in microcavity quality factors as high as $3.9 \times 10^{7}$. Single-mode lasing is achieved, and the lasing threshold depends strongly on coupling conditions. We believe that the demonstration of the compatibility of ion beam processing and high- $Q$ cavity formation will inspire a wealth of new experiments in microresonator science and technology. It implies that alternative dopants such as $\mathrm{Tb}$ or Eu can be implanted to fabricate Si-based lasers that operate in the visible range of the spectrum. Also, any nanostructure that can be made using ion implantation into $\mathrm{SiO}_{2}$ can now be incorporated into a high- $Q$ resonator on Si. Examples are implantation of noble metals $(\mathrm{Cu}, \mathrm{Ag}, \mathrm{Au})$ to form nanocrystals with well-defined surface plasmon resonance and associated non-linear properties, ${ }^{11}$ or Si implantation to form optically active Si nanocrystals that act as sensitizers for Er of show optical gain. ${ }^{12}$ Eventually this work can lead to the integration of Si-based active microresonators with optoelectronic components on a single silicon chip.

The Dutch part of this work is part of the research program of FOM, which is financially supported by NWO. The work at Caltech was funded by the NSF, DARPA and the Caltech Lee Center for Advanced Networking. Mark Brongersma is gratefully acknowledged for experiments in the early phase of this work. A.P. gratefully acknowledges the hospitality of both the Atwater and Vahala groups during his sabbatical stay at Caltech.

${ }^{1}$ D. K. Armani, T. J. Kippenberg, S. M. Spillane, and K. J. Vahala, Nature (London) 421, 925 (2003).

${ }^{2}$ L. Yang, D. K. Armani, and K. J. Vahala, Appl. Phys. Lett. 83, 825 (2003).

${ }^{3}$ A. Polman, J. Appl. Phys. 82, 1 (1997).

${ }^{4}$ M. Cai, O. Painter, and K. J. Vahala, Phys. Rev. Lett. 85, 74 (2000).

${ }^{5}$ S. M. Spillane, T. J. Kippenberg, O. J. Painter, and K. J. Vahala, Phys. Rev. Lett. 91, 043902 (2003).

${ }^{6}$ B. K. Min et al. (unpublished).

${ }^{7}$ M. Cai and K. Vahala, Opt. Lett. 26, 884 (2001).

${ }^{8}$ M. Cai, O. Painter, K. J. Vahala, and P. C. Sercel, Opt. Lett. 25, 1430 (2000).

${ }^{9}$ W. von Klitzing, E. Jahier, R. Long, F. Lissillour, V. Lefèvre-Seguin, J. Hare, J.-M. Raimond, and S. Haroche, J. Opt. B: Quantum Semiclassical Opt. 2, 204 (2000).

${ }^{10}$ M. Fujii, M. Yoshida, Y. Kanzawa, S. Hayashi, and K. Yamamoto, Appl. Phys. Lett. 71, 1198 (1997).

${ }^{11}$ F. Hache, D. Ricard, and C. Flytzanis, J. Opt. Soc. Am. B 3, 1647 (1986).

${ }^{12}$ L. Pavesi, L. Dal Negro, C. Mazzoleni, G. Franzò, and F. Priolo, Nature (London) 408, 440 (2000). 